\title{
陈运泰: 中国科技期刊的发展离不开科学家的参与和奉献
}

李亚敏, 魏建晶

\section{An interview with Prof. Yuntai Chen: Chinese scientists are the strong backing of China's scholarly journals}

Yamin Li \& Jianjing Wei

doi: 10.1360/TB-2020-1421

陈运泰, 地球物理学家, 1940年出生于厦门, 1962年毕 业于北京大学地球物理系, 1966年(研究生)毕业于中国科学 院地球物理研究所. 中国科学院大学荣誉讲席教授, 中国 科学院院士, 发展中国家科学院院士, 研究成果获多项国 际、国家奖励. 在中国科技期刊国际影响力提升计划、登 峰行动计划、卓越行动计划等项目中担任评委或监察委员, 1988 2012年担任《中国科学》《科学通报》(以下简称“两 刊”)联合编委会编委, 长期关注中国科技期刊特别是 “两 刊”的发展.

值“两刊”创刊70周年之际, 我们专访了陈运泰院士, 请他谈谈对“两刊”及中国科技期刊的看法.

\section{如何评价一本科技期刊是否“优秀”？}

国家有关部门评审科技期刊的指标, 对刊物发展具 有导向性作用. 您多次在中国科学技术协会组织的中国 科技期刊项目中担任评委, 您认为对科技期刊的评价标 准是什么? 如何评价一本科技期刊是否“优秀”?

陈运泰：评价科技期刊是一个难题. 简单用某种特定 的指标定量衡量期刊是一个相对省事的办法, 但可能并不 准确. 我在参与期刊项目评审工作中, 看到期刊编辑很辛 苦很敬业地对待期刊评审, 实际资助金额可能并不大, 但 是大家为了项目评审答辩, 花费很长时间进行准备, 我很 感动、很佩服. 我理解期刊编辑的心情, 毕竟能否通过评 审、能否得到资助和支持, 对期刊发展还是很重要的. 近 几年, 期刊项目评审主要是从学术指标和专家评价两个方 面考察期刊. 学术指标主要是影响因子、发文量等客观数 据, 而专家评价更多的是从期刊内容、口碑等主观感受上 衡量和评价.

科技期刊是学术研究成果传播、交流和存储的主要载 体, 是科研成果产出的具体表现形式, 所以评价科技期刊 优劣最主要的是看它刊载的成果是否优秀. 如果一份科技 期刊刊载的论文是原创的、前沿的成果, 对学科发展有较 大促进作用, 能引起领域内科学家的兴趣并深人探究, 那

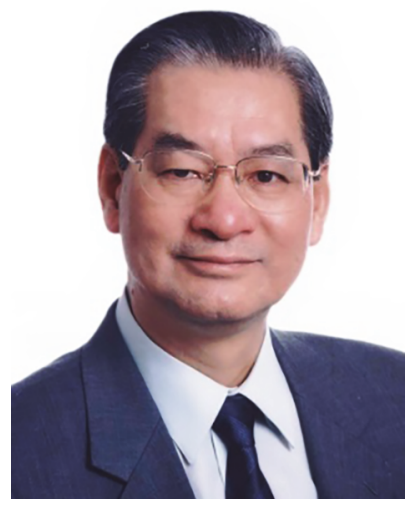

陈运泰 院士

么这份期刊就是好的期刊; 反之, 如果一份期刊翻阅开来 没有什么值得借鉴的, 都是人云亦云、追着别人跑的工作, 无法给科学家以学术触动, 那么这份期刊就很难说是优秀 的期刊.

科技期刊最重要的是刊载好的科研成果, 那么您认 为什么样的论文才是好的科研成果?

陈运泰：毛主席曾说过：“人类总是不断地总结经验, 有所发现，有所发明，有所创造，有所前进.”我觉得这4 个“有所”很适用于对科研成果和学术论文的评价. 在概念 上有突破、在方法上有创新, 以及在结论上有推进, 这样 的成果才能称之为优秀成果.

当然, 优秀的成果还要能经受住时间的检验. 有些不 符合主流观念、不符合一般认识的成果可能在最初并不受 人待见, 在若干年之后才被证明是正确的. 从科学发展历 史看, 优秀的成果发表在一般刊物上也很常见，例如有关 “板块构造理论”的一些具有里程碑意义的论文在提出之初 因为不符合当时的主流思想, 很难在大刊上获准发表, 只 能发表在一般刊物上, 而如今与板块构造理论相悖的论文 也不容易在大刊上发表了。此一时彼一时, 这也符合科学 理论的发展规律. 


\section{中国科学家要有“期刊因我而出名”的精神}

不可否认, 目前中国的科技期刊整体还不够强, 还 没有达到它应有的高度或者说还没有满足中国科学家 的期望. 很多中国科学家不愿意将他们最好的科研成 果发表在中国的期刊上, 您怎样看待这种现象?

陈运泰: 我个人认为, 期刊与论文的关系要搞清楚, 先有好的论文才有好的期刊; 论文好, 发表在哪里都是好 论文. 刊载好论文的期刊是好的期刊, 但是反过来并不成 立一一好期刊上刊载的论文未必都是好论文. 好成果虽然 没有发表在国际大刊上, 但真正有创新的成果, 经过实践 和时间检验的成果, 发表在什么期刊上, 并不影响他们最 终获得世界的认可. 屠呦呦教授获得诺贝尔奖的代表作品 发表在中文期刊《科学通报》上就是很好的例子.

前面说了很多关于期刊的评价工作, 其实中国科技期 刊的发展最后还是要 “落脚”于中国的整体科技实力. 如果 中国的科研水平落后, 中国的科学家没有话语权, 中国的 科技期刊也很难有好的发展. 编辑的努力当然不可缺少, 但最重要的还是要依靠中国的科学家和中国的基础科研 水平, 这不是仅靠编辑努力就能提升的事情, 还需要广大 科学家的行动.

著名数学家许宝騄教授曾说过: “我不希望自己的文章 因登在有名的杂志上而出名, 我希望杂志因为登了我的文 章而出名.” 我很欣赏这段话. 我认为, 中国科学家发表论 文, 不能追求 “我借期刊而出名”, 而要有 “期刊因我而出 名” 的精神, 在一本期刊还不具备特别大的名气时, 把优 秀的成果投给它, 需要一种奉献和牺牲精神. 中国这样一 个科技大国确实需要有几份在国际学术传播领域具有引 领性的科技期刊. 为了提升中国的科技期刊竞争力, 可能 在发展初期需要中国科学家作出奉献, 要将他们最优、最 前沿的重大成果, 对学科发展有重大意义和影响的成果, 贡献给国内的期刊, 以促进中国科技期刊在国际学术圈和 科技出版界的影响力. 一旦这个影响力建立起来, 进人良 性循环, 期刊的后续发展就主动得多, 中国的科学家也有 了更好的发声平台.

目前正在落实的“代表作”科研评价制度, 强调成果 本身且在政策上鼓励在中国期刊上发表代表作。在稿 源竞争激烈的情况下, 中国科技期刊如何能够拿到中 国科学家最优秀的成果?

陈运泰: 国内很多科研单位的成果评价多年来有形 无形中都是更推崇国际大刊, 中国期刊想拿到中国科学 家最优秀的成果确实很不容易. 尽管目前有政策上的助 力, 例如院士候选人需要在以“两刊”为代表的国内优秀期 刊上发表代表作等. 这种改革的初衷是促进中国期刊地 位的提升, 对于成果高产的科学家而言, 在他众多成果中 奉献出一两篇给国内期刊问题不大, 但对成果少者来说
可能就不容易.

需要注意的是, 刊物的发展单依靠政策是不够的, 还 得靠科学家转变思路, 发挥情怀, 贡献最优的成果来支持 国内期刊的发展. 此外, 不能单方面要求科学家讲情怀, 还需要给他们提供讲情怀的土壤. 例如, 不能一方面要求 他们贡献优秀论文给国内期刊, 却在各种奖项评比时还唯 国际大刊马首是瞻. 目前科研成果评价制度正在进行改 革, 希望今后能够调动中国科学家的积极性, 参与到包括 “两刊”在内的中国科技期刊的发展中.

以刊载中国科学家成果为主的中国科技期刊水平 明显受到中国科研发展水平的影响, 您怎么看待中国 目前的科研环境和科学家的工作精神?

陈运泰: 与几十年前相比, 中国当下的科研条件改善 很多, 各种软硬件都很好了, 这一点从每年国家对科研经 费的投人就可以看出来. 但是现在真正能立于世界之林的 科研成果不多. 总体感觉, 现在有些青年科研工作者在科 研上投入的精力不是很多, 干劲也不够大, 距离 “你追我赶” 的科研状态还差一些, 这可能与杂事多、心思较分散有关.

有一种说法, 看一个单位怎么样就看他们办公大楼晚 上的灯光. 如果晚上灯火通明, 大家都夜以继日的努力工 作, 那这个单位很可能实力强、成果多; 反之, 如果一个单 位无人加班, 那么这个单位很难有多少亮眼的成绩. 要产 出具有国际水平的科研成果需要有“国际水平的干劲”, 不 加班加点的努力付出, 不可能有领先的成果, 也只能在人 家后面跟跑.

科研工作者一定要有持续的科研干劲和科研热情. 我 记得2009年发表在《中国科学 D辑: 地球科学》上的那篇“地 震预测：回顾与展望”的文章, 当时写作成文只用了 4 天时 间; 但是, 在动笔之前, 确是经过了长时间地阅读文献、调 研和思考. 科研工作, 就是需要付出时间和精力, 前期基 础做踏实了, 后期才可能高效地整理成文.

\section{寄语“两刊”}

除了稿源上注重择优录用, 刊载对学科发展有推 动、对科学家有触动的优秀成果之外, 您觉得期刊编辑 还需要注重哪些方面的工作?

陈运泰: 期刊工作前前后后包含多个方面, 从组稿、 质量评审到编辑加工和后续宣传等. 首先, 从内容上抓到 优质的成果, 从评审和出版上精益求精, 获得专业人员的 认可, 这是首要和核心的工作. 其次还要重视期刊宣传工 作. 有些期刊办得不错, 但是办刊的编辑可能只顾埋头干 活, 不管外面的事情; 有些编辑可能就比较活跃, 期刊知 名度高. 各方面都办得好, “酒香不怕巷子深”的期刊确实 也有; 但如果在其他条件都相同的情况下, 具有更活跃编 辑的期刊相对更容易在评审中胜出, 更容易被更多读者关 注. 因此, 除了编辑出版工作要做好之外, 也要重视期刊 
的宣传工作.

除上述两点外, 还需要注意文字润色工作. 有时候科 学家会因为其本人过于熟悉论文的内容而不知不觉地在 表达上过于简化和缩略, 编辑加工时应协助作者进行完整 的表达; 或者有个别作者的文字表述有不足, 特别是外语 表达, 期刊可以帮助作者进行润色. 好的语言表达有助于 更准确的描述和更广泛的交流.

近几年中国创办新刊的热情高涨, 您觉得“两刊”所 处环境与以前相比有什么变化?

陈运泰: 现在的新刊很多, 而且许多新刊也办得很好, 这对“两刊” 是一个挑战. “两刊”过去在中国科学家心目中 的地位很高, “两刊”要刊登最优秀、最新的科技成果, 这是 我们关心 “两刊”发展的人的共识和心声. 新形势下, “两 刊” 想在中国科学家心目中继续保持领头羊的地位, 要比 以往更辛苦. “两刊”评审很严、出版质量很高, 这是“两刊”
一直以来在国人心中的口碑, 这是有利的方面. 但在目前 的形势下, “两刊”要上升到很高的地位, 难度很大, 需要付 出更多的努力. 专业的刊物面向专业内的人, 专业领域的 人认可的才是好刊物; 综合性的刊物面向更多的受众, 这 是两回事. 我个人认为“两刊”的涵盖面太广, 对专业科研 人员来说, 翻阅起来不如专业刊物方便. 专业刊物中刊载 的基本都是与自己领域相关的成果，而“两刊”因为包罗万 象, 可能要翻阅好几期才能看到几篇能用得到的文章, 我 认为这是不利的一面. 但是现在已经是互联网时代, 大家 不需要再去翻阅厚重的纸质刊, 通过网络就可以搜索到相 关主题的文章, 如果做到便捷地下载阅读, 不利的方面也 就被弱化了.

最后，我希望“两刊”能够坚守初心，在新的时代发展 得更好! 也相信在我们敬业又专业的编辑团队的努力及中 国科学家的支持下, “两刊”能够做到这一点! 